\title{
A FIXED POINT APPROACH TO HOMOLOGICAL PERTURBATION THEORY
}

\author{
DONALD W. BARNES AND LARRY A. LAMBE
}

(Communicated by Frederick R. Cohen)

\begin{abstract}
We show that the problem addressed by classical homological perturbation theory can be reformulated as a fixed point problem leading to new insights into the nature of its solutions. We show, under mild conditions, that the solution is essentially unique.
\end{abstract}

\section{INTRODUCTION}

It has proved useful to have theorems that address when a subcomplex $M$ of a given chain complex $A$ can be changed in a way that reflects a change in $A$ and preserves the inclusion. Specifically, when the subcomplex is a retract, it is often useful to be able to transfer a change in the differential of $A$ to one in $M$ in a way that preserves the retraction condition. This is far from trivial; in fact, the Basic Perturbation Lemma [B, S, G] provides a solution to a particular instance of this problem in the case that $M$ is actually a strong deformation retract of $A$. We recall that

$$
(M \underset{f}{\stackrel{\nabla}{\rightleftarrows}} A, \phi)
$$

is Strong Deformation Retraction data (SDR-data) if and only if

(i) $f \nabla=1_{M}$,

(ii) $\nabla f=1_{A}-D(\phi)$,

where $D(\phi)=d \phi+\phi d$. It was shown in [LS] that the additional conditions:

(iii) $\phi \phi=0$,

(iv) $\phi \nabla=0$,

(v) $f \phi=0$

could be assumed by replacing $\phi$ by $D(\phi) \phi D(\phi) d D(\phi) \phi D(\phi)$ if necessary.

The Basic Perturbation Lemma is usually stated in terms of filtered SDR-data, i.e., data for which there are filtrations for $M$ and $A$ and for which the maps

Received by the editors December 6, 1989.

1980 Mathematics Subject Classification (1985 Revision). Primary 55U25, 18 G35.

Key words and phrases. Chain homotopy, homological perturbation, fixed point, iterative methods.

This research was supported by a grant from the Australian Research Council. 
are filtration preserving. With these hypotheses and the additional hypothesis that $t: A \rightarrow A$ is a differential change in $A$, i.e., $(d+t)^{2}=0$, such that $\phi t$ lowers filtration, the lemma states that there are perturbations

$$
\begin{array}{ll}
f_{\infty}=f-f \Sigma_{\infty} \phi, & \nabla_{\infty}=\nabla-\phi \Sigma_{\infty} \nabla, \\
\partial_{\infty}=d_{M}+f \Sigma_{\infty} \nabla, & \phi_{\infty}=\phi-\phi \Sigma_{\infty} \phi,
\end{array}
$$

where

$$
\Sigma_{\infty}=t-t \phi t+\cdots+(-t \phi)^{n} t+\cdots,
$$

giving rise to new SDR-data

$$
\left(\left(M, \partial_{\infty}\right) \underset{f_{\infty}}{\stackrel{\nabla_{\infty}}{\rightleftarrows}}(A, d+t), \phi_{\infty}\right)
$$

satisfying (i)-(v). The differential change $t$ has been termed the initiator in [GLS1, GLS2]. An application to the Hirsch complex was given in [B]. There is an application to fibrations in [G] and to iterated fibrations in [LS]. An application to a class of resolutions was given in [L] and to distinguished resolutions in [GL]. Results concerning the preservation of algebra and co-algebra structure are given in [GLS1, GLS2], and in [HK] where it is also shown that similar formulas exist for the transference of a change in the differential in $A$ in the more general setting where one only has $f \nabla$ homotopic to the identity. This is done by using the mapping cylinder to reduce the more general problem to a simple application of the Basic Perturbation Lemma. Since all of these applications depend crucially on the Basic Perturbation Lemma, we are interested in investigating the status of the particular solution that the lemma provides in terms of the following general transference problem.

Transference Problem (SDR-version). Given SDR-data

$$
(M \underset{f}{\stackrel{\nabla}{\rightleftarrows}} A, \phi)
$$

and a change $t$ in the differential on $A$, so that $\xi^{2}=0$ where $\xi=d+t$, find new SDR-data

$$
\left(\left(M, d^{\prime}\right) \underset{f^{\prime}}{\stackrel{\nabla^{\prime}}{\rightleftarrows}}(A, \xi), \phi^{\prime}\right) .
$$

In the next section we rephrase the transference problem in a way that led us to new insights. The development follows in $\S \S 3,4$. In $\S 5$, we will show that the maps of the Basic Perturbation Lemma are essentially unique under the conditions stated there. We will give applications in $\S 6$. In this paper, all modules will be graded differential modules over a commutative ring $R$ with 1 .

\section{SPlitTing homotopies}

First we will replace conditions $(i)-(v)$ in the definition of SDR-data by the following conditions:

(1) $\phi: A \rightarrow A$ is a morphism of degree 1 of graded $R$-modules; 
(2) $\phi^{2}=0$;

(3) $\phi d \phi=\phi$.

We claim that (1)-(3) are equivalent to (i)-(v). To see that, suppose (i)-(v) hold. Applying $\phi$ to (ii) and using the other identities shows that $\phi d \phi=\phi$. Conversely, let $\pi=1_{A}-D(\phi)$ and suppose that (1)-(3) hold. Another easy computation shows that $\pi^{2}=\pi$ so $\pi$ is a projection and hence we have a splitting

$$
A=\operatorname{im}(\pi) \oplus \operatorname{ker}(\pi) .
$$

Let $M=\operatorname{im}(\pi)$ and $(M \underset{f}{\stackrel{\nabla}{\rightleftarrows}} A, \phi)$ be defined by taking $\nabla$ to be the inclusion and $f$ to be the corestriction of $\pi$. It is an easy exercise to verify (i)-(v).

Note that we have changed the sign of the homotopy from the usual convention in homological perturbation theory. This introduces alternating signs in the formulae for the limit SDR-data in the Basic Perturbation Lemma, but it is more convenient for our purposes.

Definition. We will call any $\phi$ satisfying (1)-(3) a splitting homotopy.

We will generally use the notation $D_{\tau}(\mu)$ for the graded commutator $\tau \mu-$ $(-1)^{|\mu|} \mu \tau$ for a degree -1 map $\tau$ in what follows.

The transference problem, translated into an equivalent formulation using the notion of splitting homotopies, becomes:

Transference Problem. Given a splitting homotopy $\phi:(A, d) \rightarrow(A, d)$ and a new differential $\xi$, find a splitting homotopy $\phi^{\prime}:(A, \xi) \rightarrow(A, \xi)$ such that

$$
\operatorname{im}(\pi) \cong_{R} \operatorname{im}\left(\pi^{\prime}\right) \quad \text { (isomorphic as } R \text { - modules, ignoring differentials), }
$$

where $\pi=1-D_{d}(\phi)$ and $\pi^{\prime}=1-D_{\xi}\left(\phi^{\prime}\right)$.

\section{FORMAL SOLUTIONS TO THE FIXED POINT PROBLEM}

Note that any solution $\phi^{\prime}$ is a fixed point of the function $F$ : $\operatorname{End}(A) \rightarrow$ $\operatorname{End}(A)$ given by $F(x)=x \xi x$. Consider that fixed point problem generally. Any nonzero solution is an $R$-module map $x: A \rightarrow A$ of degree 1 since $\xi$ has degree -1 . For a systematic solution, we must construct our fixed point from the given maps, i.e., $d, t, \phi(\xi=d+t)$. We are thus led to consider the ring

$$
\mathscr{P}(R)=R[d, t, \phi] / I,
$$

where $R[d, t, \phi]$ is the ring of noncommutative polynomials in the indeterminates $d, t, \phi$ of degrees $-1,-1,1$ respectively, over the base ring $R$ and $I$ is the ideal generated by the relations

$$
\begin{aligned}
\phi d \phi & =\phi, & & \phi^{2}=0, \\
d^{2} & =0, & \xi^{2} & =0
\end{aligned}
$$

and look for a solution in that ring or in its completion $\widehat{\mathscr{P}}(R)$ (the formal power series ring). 
The elements in the power series ring having degree 1 are all of the form $x=\sum_{n=0}^{\infty} x_{n}(\phi t)^{n} \phi$, where $x_{i} \in R$, so we seek a solution of this form. For such $x$ we have

$$
F(x)=\sum_{m=0}^{\infty} \sum_{n=0}^{\infty} x_{m}(\phi t)^{m} \phi \xi x_{n}(\phi t)^{n} \phi=\sum_{m=0}^{\infty} \sum_{n=0}^{\infty} x_{m} x_{n}(\phi t)^{m} \phi \xi \phi(t \phi)^{n} .
$$

Since $\phi \xi \phi=\phi+\phi t \phi$,

$$
\begin{aligned}
F(x) & =\sum_{m=0}^{\infty} \sum_{n=0}^{\infty} x_{m} x_{n}\left\{(\phi t)^{m+n}+(\phi t)^{m+n+1}\right\} \phi \\
& =\sum_{n=0}^{\infty}\left\{\sum_{i=0}^{n}\left(x_{i} x_{n-i}+x_{i} x_{n-i-1}\right)\right\}(\phi t)^{n} \phi .
\end{aligned}
$$

We thus obtain the recurrence relation

$$
\begin{aligned}
x_{n} & =\left(x_{0} x_{n}+x_{1} x_{n-1}+\cdots+x_{n} x_{0}\right)+\left(x_{0} x_{n-1}+x_{1} x_{n-2}+\cdots+x_{n-1} x_{0}\right) \\
& =2 x_{0} x_{n}+\left(x_{1} x_{n-1}+\cdots+x_{n-1} x_{1}\right)+\left(x_{0} x_{n-1}+\cdots+x_{n-1} x_{0}\right) ;
\end{aligned}
$$

i.e.,

$$
\left(1-2 x_{0}\right) x_{n}=\left(x_{1}+x_{0}\right) x_{n-1}+\left(x_{2}+x_{1}\right) x_{n-2}+\cdots+\left(x_{n-1}+x_{n-2}\right) x_{1}+x_{n-1} x_{0} .
$$

Solving

$$
\begin{array}{rlrl}
x_{0} & =x_{0} x_{0}, & & \text { so } x_{0}=1, \\
-x_{1} & =x_{0} x_{0}, & & \text { so } x_{1}=-1, \\
\vdots & & \vdots \\
-x_{n} & =x_{n-1} x_{0}, & & \text { so } x_{n}=(-1)^{n} .
\end{array}
$$

These equations show that $x=\sum_{n=0}^{\infty}(-1)^{n}(\phi t)^{n} \phi$ is the only nonzero solution in the power series ring.

It is tempting to use the standard iterative method for solving fixed point problems as given, for example, in [C, p. 358ff] to see how solutions might otherwise be found. We want a result, however, that will be ring independent and hence we are led to work over $\mathscr{P}(\mathbb{Z})$. We begin with initial point $\phi$ itself and apply the function $F$ repeatedly. Thus

$$
\begin{aligned}
F(\phi) & =\phi \xi \phi=\phi+\phi t \phi \\
& =(1+\phi t) \phi=\phi(1+t \phi), \\
F^{2}(\phi) & =(1+\phi t) \phi \xi \phi(1+t \phi)=(1+\phi t)^{3} \phi, \\
& \vdots \\
F^{n}(\phi) & =(1+\phi t)^{2^{n}-1} \phi=\phi(1+t \phi)^{2^{n}-1} .
\end{aligned}
$$

The term of degree $k$ in $F^{n}(\phi)$ has coefficient

$$
c_{k}^{n}=\left(\begin{array}{c}
2^{n}-1 \\
k
\end{array}\right)=\frac{\left(2^{n}-1\right)\left(2^{n}-2\right) \cdots\left(2^{n}-k\right)}{1 \cdot 2 \cdots k}
$$


for $k \leq 2^{n}-1$. Let $2^{t}$ be the highest power of 2 which does not exceed $k$. Let $2^{s}$ be the highest power of 2 dividing $r$. Then

$$
\frac{2^{n}-r}{r}=\frac{2^{n-s}-r / 2^{s}}{r / 2^{s}} \equiv-1 \quad \bmod 2^{n-s} \text {. }
$$

Since for $r \leq k$ we have $s \leq t$, it follows that $c_{k}^{n} \equiv(-1)^{k} \bmod 2^{n-t}$. Thus in the 2-adic valuation on $\mathbb{Z}, \lim _{n \rightarrow \infty} c_{k}^{n}=(-1)^{k}$. This limit using the 2-adic valuation gives us the classic power series solution from the Basic Perturbation Lemma:

$$
F^{\infty}(\phi)=\phi-\phi t \phi+(\phi t)^{2} \phi+\cdots
$$

To see what happens when we choose a different starting point for the iteration, we denote the above power series by $\psi$. For the power series $x=\sum x_{i}(\phi t)^{i} \phi$, we put $\bar{x}=\sum x_{i}(\phi t)^{i}$ and $\underline{x}=\sum x_{i}(t \phi)^{i}$. We then have $x=\bar{x} \phi=\phi \underline{x}$ and

$$
\begin{aligned}
F(x) & =\bar{x} \phi \xi \phi \underline{x}=\bar{x}(\phi+\phi t \phi) \underline{x} \\
& =(1+\phi t) \bar{x} \bar{x} \phi=\phi \underline{x} \underline{x}(1+t \phi) .
\end{aligned}
$$

Suppose we start the iteration at $\psi+x^{[0]}$. Define $x^{[r+1]}=F\left(\psi+x^{[r]}\right)-\psi$. We have

$$
\begin{aligned}
x^{[r+1]} & =F\left(\psi+x^{[r]}\right)-\psi=(1+\phi t)\left(\bar{\psi}+\bar{x}^{[r]}\right)^{2} \phi-\psi \\
& =(1+\phi t)\left(2 \bar{\psi} \bar{x}^{[r]}+\bar{x}^{[r]} \bar{x}^{[r]}\right) \phi .
\end{aligned}
$$

If 2 divides $x_{0}^{[r]}, x_{1}^{[r]}, \ldots, x_{k-1}^{[r]}$, then 2 divides $x_{k}^{[r+1]}$, and if $2^{\alpha}$ divides $x_{0}^{[r]}, x_{1}^{[r]}, \ldots, x_{k}^{[r]}, \alpha \geq 1$, then $2^{\alpha+1}$ divides $x_{k}^{[r+1]}$. Thus $2^{r-k}$ divides $x_{k}^{[r]}$ provided 2 divides $x_{0}^{[0]}$. Consequently, in the 2 -adic valuation on $\mathbb{Z}$, we then have $\lim _{r \rightarrow \infty} x_{k}^{[r]}=0$ and the iteration converges to $\psi$. If, however, we start the iteration at a point $y$ with 2 dividing $y_{0}$, it is easily seen that the iteration converges to the limit 0 .

\section{THE PERTURBATION ALGEBRA}

We have found it useful to introduce the algebra $\mathscr{P}(\mathbb{Z})$. Any particular perturbation problem involves a homomorphism of this algebra into the ring of endomorphisms of the module in question. Because of this central rôle, we call $\mathscr{P}=\mathscr{P}(\mathbb{Z})$ the perturbation algebra. Given a specific transference problem on $(M \underset{\beta}{\stackrel{\alpha}{\rightleftarrows}} A, \nu)$ for modules over $R$ with respect to the initiator $\tau$, or equivalently, given a splitting homotopy $\nu: A \rightarrow A$, we have the associated representation $\rho: \mathscr{P} \rightarrow \operatorname{End}(A)$ given by $d \mapsto d_{A}, t \mapsto \tau$, and $\phi \mapsto \nu \quad(z \mapsto z \cdot 1$ for $z \in \mathbb{Z}, 1 \in R)$. The classic solution extends $\rho$ to the power series discussed above. Looking once more at the classic solution, $\phi_{\infty}=\sum_{n=0}^{\infty}(-1)^{n}(\phi t)^{n} \phi$, we see that, formally, $\phi_{\infty}=(1+\phi t)^{-1} \phi$ or $(1+\phi t) \phi_{\infty}=\phi$. This last equation can be interpreted via $\rho$ as the equation $(1+\nu \tau) \nu^{\prime}=\nu$ for the new splitting homotopy $\nu^{\prime}$. 
Proposition. Suppose $(M \underset{\beta}{\stackrel{\alpha}{\rightleftarrows}} A, \nu)$ is SDR-data satisfying (1)-(3) and $(1+\nu \tau) \nu^{\prime}=\nu$ and $\nu \tau \nu^{\prime}=\nu^{\prime} \tau \nu$. Then

(1) $\nu^{\prime}$ is a splitting homotopy with respect to $\xi=(d+\tau)$.

(2) $\nu^{\prime}$ satisfies the isomorphism condition in the statement of the Transference Problem.

Let $\pi=\beta \alpha=1-D_{d}(\nu)$ and identify $M$ with $\operatorname{im}(\pi)$ via the splitting homotopy $\nu$. Let $\pi^{\prime}=1-D_{\xi}\left(\nu^{\prime}\right)$. Then

(3) we obtain new SDR-data $\left((M, \tilde{\partial}) \underset{\tilde{\beta}}{\stackrel{\tilde{\alpha}}{\rightleftarrows}}(A, \xi), \nu^{\prime}\right)$ where

$$
\begin{aligned}
& \tilde{\partial}=d_{M}+\beta \tau \alpha-\beta \xi \nu^{\prime} \xi \alpha, \\
& \tilde{\alpha}=\alpha-\nu^{\prime} \xi \alpha, \\
& \tilde{\beta}=\beta-\beta \xi \nu^{\prime} .
\end{aligned}
$$

Proof. We have $\nu^{\prime}=\nu-\nu t \nu^{\prime}=\nu-\nu^{\prime} t \nu$ so that $\nu \nu^{\prime}=0$ and $\nu^{\prime} \nu=0$. Furthermore, $\nu^{\prime} \nu^{\prime}=\left(1-\nu^{\prime} t\right) \nu \nu\left(1-t \nu^{\prime}\right)=0$. Another easy calculation gives that $\nu^{\prime} \xi \nu^{\prime}=\nu^{\prime}$ so that $\nu^{\prime}$ is a splitting homotopy and $\pi^{\prime}$ is a projection. Now note that $\beta \nu^{\prime}=\beta \nu\left(1-t \nu^{\prime}\right)=0$ and $\nu^{\prime} \alpha=\left(1-t \nu^{\prime}\right) \nu \alpha=0$. Thus, $\pi \nu^{\prime}=$ $\alpha \beta \nu^{\prime}=0$ and $\nu^{\prime} \pi=\nu^{\prime} \alpha \beta=0$. From this we have $\pi \pi^{\prime} \pi=\pi\left(1-\xi \nu^{\prime}-\nu^{\prime} \xi\right)=\pi$, and similarly defining $M^{\prime}=\operatorname{im}\left(\pi^{\prime}\right)$ and maps $\alpha^{\prime}$ and $\beta^{\prime}$ so that we have SDRdata $\left(M^{\prime} \underset{\beta^{\prime}}{\stackrel{\alpha^{\prime}}{\rightleftarrows}} A, \nu^{\prime}\right)$, we have $\beta^{\prime} \nu=0, \nu \alpha^{\prime}=0, \pi^{\prime} \nu=0, \nu \pi^{\prime}=0$, and hence $\pi^{\prime} \pi \pi^{\prime}=\pi^{\prime}$. Thus $\tilde{\pi}=\left.\pi\right|_{\operatorname{im}\left(\pi^{\prime}\right)}$, and $\tilde{\pi}^{\prime}=\left.\pi^{\prime}\right|_{\operatorname{im}(\pi)}$ are mutually inverse $R$ module isomorphisms, $M^{\prime}=\operatorname{im}\left(\pi^{\prime}\right) \cong_{R} \operatorname{im}(\pi)=M$. We may therefore define $\tilde{\partial}$ on $M$ by $\tilde{\partial}=\pi \xi \tilde{\pi}^{\prime}$. Thus, if we define $\tilde{\alpha}=\tilde{\pi}^{\prime}$ and $\tilde{\beta}=\pi \pi^{\prime}$, we have $\tilde{\partial} \tilde{\beta}=\pi \xi \pi^{\prime} \pi \pi=\pi \xi \pi^{\prime}=\pi \pi^{\prime} \xi=\tilde{\beta} \xi$ and $\tilde{\alpha} \tilde{\partial}=\pi^{\prime} \pi \xi \pi^{\prime}=\pi^{\prime} \pi \pi \xi=\xi \pi^{\prime}=\xi \tilde{\alpha}$. It is easy to verify that $\tilde{\beta} \tilde{\alpha}=1$ and $\tilde{\alpha} \tilde{\beta}=\pi^{\prime}$. It also follows easily that $\tilde{\alpha}=\pi^{\prime} \alpha$ and $\tilde{\beta}=\beta \pi^{\prime}$ and hence we obtain the expressions for $\tilde{\alpha}$ and $\tilde{\beta}$ in the statement of the proposition. We also have $\tilde{\partial}=\tilde{\beta} \tilde{\alpha} \tilde{\partial}=\tilde{\beta} \tilde{\partial} \tilde{\alpha}=\beta \pi^{\prime} \xi \pi^{\prime} \alpha$, but another easy calculation shows that $\pi^{\prime} \xi \pi^{\prime}=\xi-\xi \nu^{\prime} \xi$ and so the proof is complete.

Our point is that we do not need to know that the power series for the classic solution converges, but need only some of the formal algebraic properties it satisfies in order to achieve a complete and satisfactory solution to the transference problem. If $(1+\nu \tau)$ or $(1+\tau \nu)$ is invertible in $\operatorname{End}(A)$, then we obtain a particular solution to the fixed point problem. These conditions which are conceivably stronger than the hypotheses of the theorem are conceivably still weaker than the convergence of the classic power series.

\section{UNIQUENESS QUESTIONS}

In the following discussion, splittings of several kinds will be encountered and it will be convenient to use matrix notation for various maps of the objects 
involved. We will often write

$$
f=\left(\begin{array}{ccc}
f_{11} & \cdots & f_{1 n} \\
\vdots & \ddots & \vdots \\
f_{n 1} & \cdots & f_{n m}
\end{array}\right)
$$

for a map $f: X \rightarrow Y$ where we are given direct sum decompositions $X=$ $\bigoplus_{j=1}^{m} X_{j}$ and $Y=\bigoplus_{j=1}^{n} Y_{j}$, with inclusions and projections inc ${ }_{j}^{X}$ and pro $_{j}^{X}$ for $X$ and similarly labelled inclusions and projections for $Y$, and we have written $f_{r s}=\operatorname{pro}_{r}^{Y} f$ inc $_{s}^{X}$.

Suppose that $\phi: A \rightarrow A$ is a splitting homotopy and $\xi$ is a new differential on $A$. Let $F(x)=x \xi x$, as before. If $\phi^{\prime}$ is a fixed point of $F$ and $P: A \rightarrow A$ is an invertible chain map with respect to $\xi$, then $F\left(P^{-1} \phi^{\prime} P\right)=P^{-1} \phi^{\prime} P \xi P^{-1} \phi^{\prime} P=$ $P^{-1} \phi^{\prime} P$ so that the conjugate is another fixed point. If $\phi^{\prime}$ satisfies the isomorphism condition, then so does $P^{-1} \phi^{\prime} P$. Thus for solutions to the transference problem, the most we can hope for is uniqueness up to conjugacy. We shall show that this in fact holds subject to some conditions on $R$ and $A$. Our argument is a modification of the familiar proof that an acyclic free complex over a PID has a contracting homotopy. We begin with the presumably well-known

Lemma 1. Suppose $s_{1}$ and $s_{2}$ are contracting homotopies on $K\left(H_{n}(K)=0\right.$, for all $n \geq 0$ ) and $s_{i}^{2}=0$. Then $\varphi=s_{1} d+d s_{2}$ is an invertible chain map with inverse $s_{2} d+d s_{1}$ and $s_{2}=\varphi^{-1} s_{1} \varphi$.

The proof is immediate. We remark that no assumptions on the ground ring or module structure are needed; the given contractions provide the necessary splittings.

Lemma 2. Let $K, K^{\prime}$ be chain complexes of finite type and free over $R$ with $K_{n} \cong{ }_{R} K_{n}^{\prime}$ for all $n \geq 0$, where $R$ is a PID. Suppose $H(K) \cong H\left(K^{\prime}\right)$ and that $H_{0}(K)$ is free. Then there exists a chain isomorphism $f: K \rightarrow K^{\prime}$.

Proof. Put $K_{-1}=H_{0}(K)$ and $K_{-1}^{\prime}=H_{0}\left(K^{\prime}\right)$. We define $f_{n}: K_{n} \rightarrow K_{n}^{\prime}$ inductively, starting with any isomorphism $f_{-1}: H_{0}(K) \rightarrow H_{0}\left(K^{\prime}\right)$.

Since $d K_{n}=B_{n-1}(K)$ is free, $K_{n} \cong Z_{n}(K) \oplus B_{n-1}(K)$. Choosing a complement to $Z_{n}(K)$ in $K_{n}$ defines a homomorphism $u: B_{n-1}(K) \rightarrow K_{n}$ and we then have $K_{n}=Z_{n}(K) \oplus u B_{n-1}(K)$. We suppose that isomorphisms $f_{r}: K_{r} \rightarrow K_{r}^{\prime}$ have been constructed for $r<n$ with $d f_{r}=f_{r-1} d$ and $f_{r}\left(B_{r}(K)\right)=B_{r}\left(K^{\prime}\right)$. Thus $B_{n-1}(K) \cong B_{n-1}\left(K^{\prime}\right)$ with the restriction of $f_{n-1}$ providing an isomorphism. But $u B_{n-1}(K) \cong B_{n-1}(K)$ and $u^{\prime} B_{n-1}\left(K^{\prime}\right) \cong B_{n-1}\left(K^{\prime}\right)$. Thus setting $f_{n} x=u^{\prime} f_{n} d x$ for $x \in u B_{n-1}(K)$ defines an isomorphism $u B_{n-1}(K) \rightarrow$ $u^{\prime} B_{n-1}\left(K^{\prime}\right)$, and $d f_{n} x=d u^{\prime} f_{n-1} d x=f_{n-1} d x$.

We still have to define $f_{n}$ on $Z_{n}(K)$, the only requirement being that it give an isomorphism $Z_{n}(K) \rightarrow Z_{n}\left(K^{\prime}\right)$ with $f_{n}\left(B_{n}(K)\right)=B_{n}\left(K^{\prime}\right)$. We know that $Z_{n}(K)$ and $Z_{n}\left(K^{\prime}\right)$ are free of the same rank. We can choose a basis $z_{1}, \ldots, z_{s}$ 
of $Z_{n}(K)$ such that $e_{1} z_{1}, \ldots, e_{s} z_{s}$ generate $B_{n}(K)$, where the $e_{i} \in R$ and $e_{i}$ divides $e_{i+1}$. The $e_{i}$ are determined up to multiplication by units. Similarly, we can choose $z_{1}^{\prime}, \ldots, z_{s}^{\prime}$. Since $H_{n}(K) \cong H_{n}\left(K^{\prime}\right)$, we have $e_{1} z_{1}^{\prime}, \ldots, e_{s} z_{s}^{\prime}$ generating $B_{n}\left(K^{\prime}\right)$. We thus define $f_{n} z_{i}=z_{i}^{\prime}$, completing the definition of $f_{n}$ and satisfying all of the requirements.

Lemma 3. Suppose $A$ is a free chain complex over the PID $R$ and that $H_{0}(A)$ is free. Suppose $\phi_{1}$ and $\phi_{2}$ are splitting homotopies on $A$ with $\operatorname{im}\left(\pi_{1}\right) \cong_{R} \operatorname{im}\left(\pi_{2}\right)$ and of finite type. Then $\operatorname{ker}\left(\pi_{1}\right)$ and $\operatorname{ker}\left(\pi_{2}\right)$ are isomorphic chain complexes.

Proof. Put $K=\operatorname{im}\left(\pi_{1}\right), L=\operatorname{ker}\left(\pi_{1}\right), K^{\prime}=\operatorname{im}\left(\pi_{2}\right)$, and $L^{\prime}=\operatorname{ker}\left(\pi_{2}\right)$. We have $A=K \oplus L=K^{\prime} \oplus L^{\prime}$. Since $L$ is free and $H(L)=0$, there exists a contracting homotopy $s: L \rightarrow L$, and we have $L_{n}=B_{n} L \oplus s\left(B_{n-1} L\right)$. Similarly $L_{n}^{\prime}=B_{n} L^{\prime} \oplus s^{\prime}\left(B_{n-1} L^{\prime}\right)$ for some contracting homotopy $s^{\prime}$ of $L^{\prime}$. By Lemma 2 , there exists a chain isomorphism $f: K \rightarrow K^{\prime}$. We shall construct inductively $R$-module isomorphisms $g_{n}: B_{n} L \rightarrow B_{n} L^{\prime}$ and $h_{n}: s\left(B_{n-1} L\right) \rightarrow s^{\prime}\left(B_{n-1} L^{\prime}\right)$ such that, for $G_{n}=g_{n} \oplus h_{n}: L_{n} \rightarrow L_{n}^{\prime}$, we have $d G_{n}=G_{n-1} d$. We start the induction by putting $L_{-1}=0=L_{-1}^{\prime}$ and $g_{-1}=h_{-1}=0$. Suppose $g_{n-1}$ and $h_{n-1}$ have been constructed. We put $h_{n}=s^{\prime} g_{n-1} d: s\left(B_{n-1} L\right) \rightarrow s^{\prime}\left(B_{n-1} L^{\prime}\right)$. Then $h_{n}$, being the composite of three isomorphisms, is an isomorphism. Now

$$
Z_{n} A=Z_{n} K \oplus B_{n} L=Z_{n} K^{\prime} \oplus B_{n} L^{\prime}
$$

is free and $Z_{n} K$ and $Z_{n} K^{\prime}$ have the same finite rank. Hence $B_{n} L$ and $B_{n} L^{\prime}$ have the same (possibly infinite) rank and there exists an isomorphism $g_{n}: B_{n} L \rightarrow B_{n} L^{\prime}$.

Now we come to the main result of this section which says that a solution to the Transference Problem is unique up to conjugacy by a chain isomorphism.

Theorem. Suppose $A$ is a free chain complex over the PID $R$ and that $H_{0} A$ is free. Suppose $\phi_{1}$ and $\phi_{2}$ are splitting homotopies on $A$ with $\operatorname{im}\left(\pi_{1}\right) \cong_{R} \operatorname{im}\left(\pi_{2}\right)$ and of finite type. Then $\phi_{1}$ and $\phi_{2}$ are conjugate.

Proof. First note that if $\phi: A \rightarrow A$ is a splitting homotopy then $\tilde{\phi}=\left.\phi\right|_{\operatorname{ker}(\pi)}$ is a contracting homotopy which squares to zero. Also note that since $\phi \pi=0$ and $\pi \phi=0$, we have $\phi=\left(\begin{array}{ll}0 & 0 \\ 0 & \phi\end{array}\right)$ in the splitting $A=\operatorname{im}(\pi) \oplus \operatorname{ker}(\pi)$. Now we are given $A=\operatorname{im}\left(\pi_{1}\right) \oplus \operatorname{ker}\left(\pi_{1}\right) \stackrel{\phi_{1}, \phi_{2}}{\rightarrow} \operatorname{im}\left(\pi_{2}\right) \oplus \operatorname{ker}\left(\pi_{2}\right)=A$ with $\operatorname{ker}\left(\pi_{i}\right)$ contractible. Thus $H\left(\operatorname{im}\left(\pi_{1}\right)\right) \cong H(A) \cong H\left(\operatorname{im}\left(\pi_{2}\right)\right)$ and by Lemma 2 , we have a chain isomorphism $\lambda: \operatorname{im}\left(\pi_{2}\right) \rightarrow \operatorname{im}\left(\pi_{1}\right)$. Also, by Lemma 3, we have a chain isomorphism $\mu: \operatorname{ker}\left(\pi_{2}\right) \rightarrow \operatorname{ker}\left(\pi_{1}\right)$. Let $\theta=\left(\begin{array}{ll}\lambda & 0 \\ 0 & \mu\end{array}\right)$ with respect to the given splittings. We have

$$
\theta^{-1} \phi_{1} \theta=\left(\begin{array}{cc}
0 & 0 \\
0 & \mu^{-1} \tilde{\phi}_{1} \mu
\end{array}\right)
$$

and hence $\tilde{\phi}_{2}$ and $\mu^{-1} \tilde{\phi}_{1} \mu$ are two contracting homotopies which square to zero on $\operatorname{ker}\left(\pi_{2}\right)$. By Lemma $1, \varphi=\mu^{-1} \tilde{\phi}_{1} \mu d+d \tilde{\phi}_{2}$ is a chain isomorphism. 
Thus

$$
\begin{aligned}
\left(\begin{array}{cc}
0 & 0 \\
0 & \tilde{\phi}_{2}
\end{array}\right) & =\left(\begin{array}{cc}
1 & 0 \\
0 & \varphi
\end{array}\right)^{-1}\left(\begin{array}{cc}
0 & 0 \\
0 & \mu^{-1} \tilde{\phi}_{1} \mu
\end{array}\right)\left(\begin{array}{cc}
1 & 0 \\
0 & \varphi
\end{array}\right) \\
& =\left(\begin{array}{cc}
1 & 0 \\
0 & \varphi^{-1}
\end{array}\right)\left(\begin{array}{cc}
\lambda^{-1} & 0 \\
0 & \mu^{-1}
\end{array}\right) \phi_{1}\left(\begin{array}{ll}
\lambda & 0 \\
0 & \mu
\end{array}\right)\left(\begin{array}{ll}
1 & 0 \\
0 & \varphi
\end{array}\right),
\end{aligned}
$$

i.e., $\phi_{2}=\left(\begin{array}{cc}\lambda & 0 \\ 0 & \mu \varphi\end{array}\right)^{-1} \phi_{1}\left(\begin{array}{cc}\lambda & 0 \\ 0 & \mu \varphi\end{array}\right)$.

We end this section with an example showing that some finiteness assumptions are necessary in the main theorem. Consider the two chain complexes $K$ and $K^{\prime}$ over $\mathbb{Z}$ where $K_{n}=0, K_{n}^{\prime}=0$ for $n \geq 4$ and $K_{0}$ is free on basis $\left\{a_{0}, a_{1}, \ldots\right\}, K_{1}$ is free on basis $\left\{b_{0}, b_{1}, \ldots\right\}, K_{2}$ is free on basis $\left\{c_{0}, c_{1}, \ldots\right\}, K_{3}$ is free on basis $\left\{d_{0}, d_{1}, \ldots\right\}$, and similarly for $K^{\prime}$ where the basis elements are denoted by the same letters with primes. Let the differential $\partial$ on $K$ be given by

$$
\partial b_{i}=a_{i}, \quad \partial c_{i}=0, \quad \partial d_{i}=c_{i},
$$

and let the differential $\partial^{\prime}$ on $K^{\prime}$ be given by

$$
\begin{gathered}
\partial^{\prime} b_{2 n}^{\prime}=a_{n}^{\prime}, \quad \partial^{\prime} b_{2 n+1}^{\prime}=0, \quad \partial^{\prime} c_{2 n}^{\prime}=0, \\
\partial^{\prime} c_{2 n+1}^{\prime}=b_{2 n+1}^{\prime}, \quad \partial^{\prime} d_{n}^{\prime}=c_{2 n}^{\prime} .
\end{gathered}
$$

Clearly, we have $Z_{2}(K)=0$, but $Z_{2}\left(K^{\prime}\right) \neq 0$ and so $K$ is not chain isomorphic to $K^{\prime}$; although they both have homology 0 in each dimension and they are isomorphic as $\mathbb{Z}$-modules in each dimension.

\section{FURTHER DISCUSSION AND EXAMPLES}

We have placed considerable emphasis on splitting homotopies which satisfy the isomorphism condition of the Transference Problem. Other sorts of transfer are possible and do occur naturally. For example, given SDR-data $(M \underset{f}{\stackrel{\nabla}{\rightleftarrows}} A, \phi)$, and a differential change $d^{\prime}=d_{M}+s$ in $M$, the obvious transfer, $\xi=\nabla d^{\prime} f$ gives a differential in $A$ for which we have SDR-data using the same maps. Most significant from our current viewpoint is that the homotopy $\phi$ is unchanged. This is because $f \nabla=1$. We may also consider more generally the case in which we have two splitting homotopies $\phi_{1}, \phi_{2}: A \rightarrow A$ with respect to a differential $\xi$ on $A$, but for which we only have $\operatorname{im}\left(\pi_{1}\right)$ homotopy equivalent to $\operatorname{im}\left(\pi_{2}\right)$ and not isomorphic as $R$-modules. In fact, this is precisely the setting referred to in $\S 1$ where we alluded to data $M \underset{g}{\stackrel{f}{\rightleftarrows}} N$ in which $f$ and $g$ are homotopy equivalences but the composites are not the identity in either direction. To see this, we follow [HK] and use the mapping cylinder, noting that background on the complexes and maps involved may be found in [HW, p. $136 \mathrm{ff}]$. Thus suppose $(\delta, M \underset{g}{\stackrel{f}{\rightleftarrows}} N, \varepsilon)$ is data such that $g f=1-D(\varepsilon)$ and 
$f g=1-D(\delta)$. Furthermore assume that $M$ and $N$ are not isomorphic as $R$-modules. Consider the mapping cylinder $N_{f}=M \oplus s M \oplus N$ where $s M$ denotes suspension, i.e., $s M_{i}=M_{i-1}$. The differential is given by

$$
d=\left(\begin{array}{ccc}
d & -1 & 0 \\
0 & -d & 0 \\
0 & f & d
\end{array}\right)
$$

We have an inclusion $j: N \rightarrow N_{f}$ given by $j=\left(\begin{array}{l}1 \\ 0 \\ 0\end{array}\right)$. The cokernel of $j$ is isomorphic to the mapping cone $\bar{N}_{f}=s M \oplus N$ with differential $\bar{d}=\left(\begin{array}{cc}-d & 0 \\ f & d\end{array}\right)$. As we have already mentioned above, it is well known that when $f$ is a homotopy equivalence, $\bar{N}_{f}$ is contractible. Given the data above, an explicit contracting homotopy may be constructed [HK]. Let $z=\left(\begin{array}{cc}0 & 0 \\ \varepsilon & f\end{array}\right)\left(\begin{array}{cc}f & 0 \\ -\delta & 0\end{array}\right)$ and $h=\left(\begin{array}{cc}\delta & g \\ 0 & -\varepsilon\end{array}\right)$. It is easy to see that $z^{2}=0$ and $D(h)=1-z$. But then $D(z)=D(1-D(h))=0$ and so $D(h+h z)=1$. Thus $\bar{h}=h+h z$ is a contracting homotopy. We want $\bar{h}$ to square to zero so replace $\bar{h}$ by $\tilde{h}=\bar{h} d \bar{h}$ if necessary. Define chain maps $\bar{N}_{f} \underset{\rho}{\stackrel{i}{\rightleftarrows}} N_{f}$ by

$$
i=\left(\begin{array}{cc}
-\delta & -g \\
1 & 0 \\
0 & 1
\end{array}\right) \quad \text { and } \rho=\left(\begin{array}{ccc}
0 & 1 & 0 \\
0 & 0 & 1
\end{array}\right)
$$

and let $\phi: N_{f} \rightarrow N_{f}$ be given by $\phi=i \tilde{h} \rho$. Then we have that

$$
D(\phi)=i D(\tilde{h}) \rho=i \rho=\left(\begin{array}{ccc}
0 & -\delta & -g \\
0 & 1 & 0 \\
0 & 0 & 1
\end{array}\right) \text {. }
$$

Thus if $\left(M \underset{k}{\stackrel{j}{\rightleftarrows}} N_{f}, \phi\right)$ is defined by $k=\left(\begin{array}{lll}1 & \delta & g\end{array}\right)$ then, recalling that $j=$ $\left(\begin{array}{l}1 \\ 0\end{array}\right)$, it is easy to see that $\phi$ is a splitting homotopy with $j k=1-D(\phi)$. Now we always have SDR-data $\left(N \underset{k_{N}}{\stackrel{j_{N}}{\rightleftarrows}} N_{f}, \phi^{\prime}\right)$ given by

$$
j_{N}=\left(\begin{array}{l}
0 \\
0 \\
1
\end{array}\right), \quad k_{N}=\left(\begin{array}{lll}
f & 0 & 1
\end{array}\right), \quad \text { and } \quad \phi^{\prime}=\left(\begin{array}{ccc}
0 & 0 & 0 \\
-1 & 0 & 0 \\
0 & 0 & 0
\end{array}\right)
$$

for the mapping cylinder and so we have two splitting homotopies $N_{f} \stackrel{\phi, \phi^{\prime}}{\longrightarrow} N_{f}$ both fixed points of $F(x)=x d x$ for $d=d_{N_{f}}$, but $M \cong \operatorname{im}(\pi)$ and $N \cong \operatorname{im}\left(\pi^{\prime}\right)$ are not isomorphic as $R$-modules by hypothesis. Having made these remarks, we will simply consider the case of two splitting homotopies $A \stackrel{\phi, \phi^{\prime}}{\longrightarrow} A$ where $\operatorname{im}(\pi)$ is homotopy equivalent to $\operatorname{im}\left(\pi^{\prime}\right)$ but not isomorphic to it as an $R$ module ignoring differentials. Let $N=\operatorname{im}(\pi)$ and $M=\operatorname{im}\left(\pi^{\prime}\right)$. Given a differential change $d^{\prime}=d_{N}+s$ in $N$, we have already noted that the differential 
in $A$ may be changed in a straightforward way to $\xi=\nabla d^{\prime} f$ with the original $\phi$ a splitting homotopy with respect to $\xi$. We may therefore consider the transference problem for $\phi^{\prime}: A \rightarrow A$ with respect to $\xi$. Any solution $\phi^{\prime \prime}$ gives rise to a new differential $d_{\infty}$ on $M$ for which we have a new homotopy equivalence $\left(M, d_{\infty}\right) \cong\left(N, d^{\prime}\right)$. In fact, we obtain a new splitting homotopy $(A, \xi) \stackrel{\phi^{\prime \prime}}{\longrightarrow}(A, \xi)$ with $\operatorname{im}\left(\pi^{\prime \prime}\right) \cong R \operatorname{im}\left(\pi^{\prime}\right)$ and by our previous results, $\phi^{\prime \prime}$ is unique up to conjugacy (subject to the conditions of the main theorem).

We now consider composite SDR-data. It was pointed out in [LS] that if

$$
\begin{aligned}
& \left(X \underset{f_{1}}{\stackrel{\nabla_{1}}{\rightleftarrows}} Y, \phi^{Y}\right), \\
& \left(Y \underset{f_{2}}{\stackrel{\nabla_{2}}{\rightleftarrows}} Z, \phi^{Z}\right)
\end{aligned}
$$

are SDR-data, then we may form a composition

$$
(X \underset{f}{\stackrel{\nabla}{\rightleftarrows}} Z, \phi)
$$

where $\nabla=\nabla_{2} \nabla_{1}, f=f_{1} f_{2}$, and $\phi=\phi^{Z}+\nabla_{2} \phi^{Y} f_{2}$.

Now suppose we change the differential $d^{Z}$ to $\xi=d^{Z}+t$ as usual. We have the classic solution

$$
\phi_{\infty}=\sum_{n=0}^{\infty}(-1)^{n}(\phi t)^{n} \phi
$$

for the new splitting homotopy $\phi_{\infty}$ of (3). But we can also handle the problem in stages, first getting the limit SDR-data for (2) and then, using the new differential $d_{\infty}^{Y}$ on $Y$, constructing new SDR-data for (1). The composite limit SDR-data solves the Transference Problem for (3). Explicitly, the new data for (2) are given by

$$
\begin{aligned}
& \phi_{\infty}^{Z}=\sum_{n=0}^{\infty}(-1)^{n}\left(\phi^{Z} t\right)^{n} \phi^{Z}, \\
& d_{\infty}^{Y}=d^{Y}+f_{2} \sum_{n=0}^{\infty}(-1)^{n} t\left(\phi^{Z} t\right)^{n} \nabla_{2} .
\end{aligned}
$$

We then use $t^{Y}=f_{2} \sum_{n=0}^{\infty}(-1)^{n} t\left(\phi^{Z} t\right)^{n} \nabla_{2}$ as an initiator for (1). Denote the new splitting homotopy for $Y$ by $\phi_{\infty}^{Y}$.

We thus have two solutions $\phi_{\infty}$ and $\phi_{\infty}^{\prime}$ to the Transference Problem:

$$
\begin{aligned}
& \phi_{\infty}=\sum_{n=0}^{\infty}(-1)^{n}(\phi t)^{n} \phi, \\
& \phi_{\infty}^{\prime}=\phi_{\infty}^{Z}+\left(\nabla_{2}\right)_{\infty} \phi_{\infty}^{Y}\left(f_{2}\right)_{\infty} .
\end{aligned}
$$

By our main theorem, (subject to its finiteness hypothesis) we may immediately conclude that these homotopies are conjugate. 


\section{REFERENCES}

[B] R. Brown, The twisted Eilenberg-Zilber theorem, Celebrazioni Archimedee del secolo XX, Simposio di topologia (1967), 34-37.

[C] R. Courant, Differential and integral calculus, Vol. 1, Blackie and Son, Glasgow, London, 1934.

[G] V. K. A. M. Gugenheim, On the chain complex of a fibration, Illinois J. Math. 16 (1972), 398-414.

[GL] V. K. A. M. Gugenheim and L. A. Lambe, Perturbation theory in differential homological algebra I, Illinois J. Math. 33 (1989), 566-582.

[GLS1] V. K. A. M. Gugenheim, L. A. Lambe, and J. D. Stasheff, Algebraic aspects of Chen's twisting cochain, Illinois J. Math. 34 (1990), 485-502.

[GLS2] __ Perturbation theory in differential homological algebra II, Illinois J. Math. (to appear).

[HW] P. J. Hilton and S. Wylie, Homology theory, Cambridge Univ. Press, Cambridge, 1960.

[HK] J. Huebschmann and T. Kadeishvili, Minimal models for chain algebras over a local ring, Math. Z. (to appear).

[L] L. A. Lambe, Resolutions via homological perturbation theory, J. Symbolic Comput. (to appear).

[LS] L. A. Lambe and J. D. Stasheff, Applications of perturbation theory to iterated fibrations, Manuscripta Math. 58 (1987), 363-376.

[S] W. Shih, Homology des espaces fibrés, Inst. des Hautes Études Sci. 13 (1962), 93-176.

Department of Pure Mathematics, University of Sydney, NSW 2006, Sydney, AusTRALIA

E-mail address, D. W. Barnes: barnes-d@math.su.oz

E-mail address, L. A. Lambe: u12117@uicvm.bitnet 